Chapter 2

\title{
Synthesis of ZnO Nanowire by MOCVD Technique: Effect of Substrate and Growth Parameter
}

\author{
Sachindra Nath Das, Jyoti Prakash Kar, \\ Junjie Xiong and Jae-Min Myoung \\ Additional information is available at the end of the chapter \\ http://dx.doi.org/10.5772/54577
}

\section{Introduction}

$\mathrm{ZnO}$ nanostructures have been studied extensively in the past few years due to their fundamental and technological importance [1-5]. In particular, it is a wide-direct-band gap ( 3.37 $\mathrm{eV})$, II-VI semiconductor with many potential applications such as nanolaser arrays $[2,6]$, gas sensors [7-10], field emission devices [11-12] and luminescent materials [13-16]. A great deal of attention has been focused on one-dimensional nanostructures because of their superior properties such as high surface-to-volume ratio, high crystalline quality, and quantum confinement effects, which are suitable for various electronics and optoelectronics applications. Up to now, many $\mathrm{ZnO}$ configurations have been reported such as nanobelts, nanowires [10-11], nanoneedles [3], nanotetrapods [9], nanocombs [17] and so on. It has also been suggested that $\mathrm{ZnO}$ nanostructures are probably the most abundant forms of any known materials. Therefore, proper control of processing parameters is essential to reproduce the desired nanostructures. Synthesis of aligned one-dimensional $\mathrm{ZnO}$ nanowire arrays has attracted much attention, which includes the techniques of metal-organic chemical vapour deposition (MOCVD), vapor-liquid-solid (VLS), pulsed laser deposition method (PLD) and solution based chemical technique [21-24]. A solution-based technique is an attractive approach for the growth of $\mathrm{ZnO}$ nanostructures because of its simplicity and low cost [25, 26]. In addition, this method allows large-scale growth of aligned $\mathrm{ZnO}$ nanowires on an arbitrary substrate, which promotes the integration of $\mathrm{ZnO}$ nanomaterials for various applications. But the main drawback of chemical process is the poor quality of nanowire. On the other hand, physical process are of particular interest since they have many advantages such as the ability to fabricate nanostructures of better quality, well controllable configurations, and good reproducibility. From a physical point of view, the synthesis methods for one-dimen- 
sional $\mathrm{ZnO}$ nanostructures are categorized into two groups, metal catalyst-assisted growth and catalyst-free growth which corresponded to vapor-liquid-solid and vapor-solid mechanisms, respectively. The latter is better than the former in terms of no metal catalytic impurity. On the other hand, MOCVD is superior because, it has abilities to produce large area; catalytic free, long, vertically aligned ZnO NWs, which have excellent morphological, structural and optical properties [27]. As the growth of $\mathrm{ZnO}$ nanowires by MOCVD is a bottomup technique, the nature of substrates has a vital role for the determination of nanowire dimension and alignment. According to lattice misfit, the most suitable substrate for $\mathrm{ZnO}$ growth is $\mathrm{ScAlMgO}_{4}$, which is expensive and technologically inconvenient [28]. Interestingly, C-plane sapphire overcomes some of the limitations. On the other hand, $\mathrm{ZnO}$ nanostructures on R-plane sapphire have an advantage for the fabrication of the next generation sensors and detectors in the form of surface acoustic wave (SAW) devices due to higher electromechanical coupling [29-31]. However, in the pursuit of next generation $\mathrm{ZnO}$-based nano devices, it would be highly preferred if well-ordered $\mathrm{ZnO}$ nanowires with high aspect ratio could be aligned onto cheap and CMOS compatible substrates, such as silicon. In addition, silicon is the most popular substrate for microelectronics and micro electro mechanical systems (MEMS), and it serves as a good integration platform. But, it is difficult to obtain wellaligned $\mathrm{ZnO}$ nanowires on silicon substrates, because the formation of an interfacial layer $\left(\mathrm{SiO}_{2}\right)$ and large lattice misfit [32,33]. On the other hand, microchannel-based solid-state devices on Si have played an important role for chemical, gas and flow sensors with compact size and superior performance [34-36]. The growth of vertically aligned $\mathrm{ZnO}$ microtubes on a silicon wafer without any catalyst are of particular interests for monolithic integration of micro/nano devices.

As far as the growth of $\mathrm{ZnO}$ nanowires on glass or $\mathrm{SiO}_{2}$ layer is concerned, deterioration in the quality and alignment of the nanowires come into picture because of the lack of specific epitaxial arrangements. In such cases the nanowires become quasi-aligned instead of vertical aligned. Since, MOCVD is a bottom up technique, the introduction of an appropriate interfacial layer may also solve the above problem. It is more preferable if the interfacial layer would be transparent and conductive for its integration with optoelectronic devices. Taking these issues into consideration, Ga doped $\mathrm{ZnO}$ (GZO) film may be a suitable interfacial layer for the growth of vertical aligned $\mathrm{ZnO}$ nanowires. In addition, $\mathrm{GZO}$ film can be used as a transparent conducting layer during the integration of the devices. Furthermore, the cost of GZO is much lower than conventionally used indium doped transparent conductive oxide layer. The nature of the substrate with a interfacial layer also take a significant role on the shape of nanowire. The control over size and morphology of nanometer and micrometer-sized semiconductor materials represents a great challenge to realizing the design of novel functional devices. Several efforts have been directed towards the synthesis of nanostructures to control the shape, aspect ratio (length/width), growth site, and growth direction. One salient feature of the $\mathrm{ZnO}$-based nanostructures is a gradual transition between different morphologies with different substrate which leads to the investigation for the effects of substrate and growth parameter on MOCVD technique. 


\section{Experimental}

$\mathrm{ZnO}$ nanowires were grown on different substrates (e.g. different types of sapphire, $\mathrm{Si}, \mathrm{Si}$ microchanel, GZO coated glass, ZnO microcreators) using MOCVD technique with diethylzinc (DEZn) as the zinc precursor. Argon and oxygen (5N) were used as carrier and reactive gases, respectively. The base pressure of the reactor chamber was $10^{-6}$ Torr. During the growth, the substrate temperature, working pressure and the nozzle-substrate spacing were fixed, but they were varied for different substrates to synthesize desired nanostructure. Morphological investigations of the nanowires were carried out using field-emission scanning electron Microscope (FESEM). The crystallinity of the nanowires was studied by X-Ray diffractometer. In addition, the microstructures of $\mathrm{ZnO}$ NWs were observed using high-resolution transmission electron microscope (HRTEM). For the cross-sectional TEM and energy dispersive X-ray (EDX) study, the samples were prepared by focused ion beam (FIB) system.

\section{Result and discussion}

\subsection{Effect of sapphire substrate}

The morphology of nanostructures greatly depends on the growth parameters as well as the underlying substrates. c-plane sapphire substrates were used for the growth of $\mathrm{ZnO}$ nanowires by MOCVD technique with substrate temperature and substrate to nozzle distance at $630{ }^{\circ} \mathrm{C}$ and $3 \mathrm{~cm}$, respectively. The experimental details are given in table 1. Fig. 1(a-c) is typical FES$\mathrm{EM}$ images of $\mathrm{ZnO}$ nanowires arrays prepared with different growth conditions. The samples constitute uniform and densely packed nanowires arrays with different surface morphologies, namely nanoneedles (Fig. 1(a)), nanonails (Fig. 1(b)) and nanowires with rounded tip (Fig. 1(c)). Cross-sectional view of the samples (inset of Fig 1.) revealed that the nanowires were grown almost perpendicularly on the substrates and their lengths were about $3 \mu \mathrm{m}$. It is known that each nucleation site usually supported growth of only one nanowires, but when the nucleation sites were close together, two nanowires combined into one with a larger diameter [37] and different surface morphologies were obtained. From XRD pattern (Fig. 2), a prominent $\mathrm{ZnO}$ (0002) peak is observed, which corresponds to the growth of $\mathrm{ZnO}$ nanowires along c-axis. No characteristic peaks indicating other impurities are observed in the samples. This is attributed to the hexagonal wurtzite structure of nanowires.

In order to investigate the structural characteristics of $\mathrm{ZnO}$ nanowires, TEM study of individual nanowires is a very important characterization technique. The bright field TEM micrographs of the $\mathrm{ZnO}$ nanowires and the corresponding HRTEM image (inset) are shown in Fig. 3. The HRTEM images indicated that the nanowires were structurally uniform and did not exhibit any noticeable defects. The HRTEMimage showed well resolved lattice with an inter-planar spacing of $0.52 \mathrm{~nm}$, which corresponds to the (0001) plane of wurtzite structure. Hence, one may infer that the nanowires possessed a single-crystal hexagonal structure with good crystalline quality along the [0001] axis. 

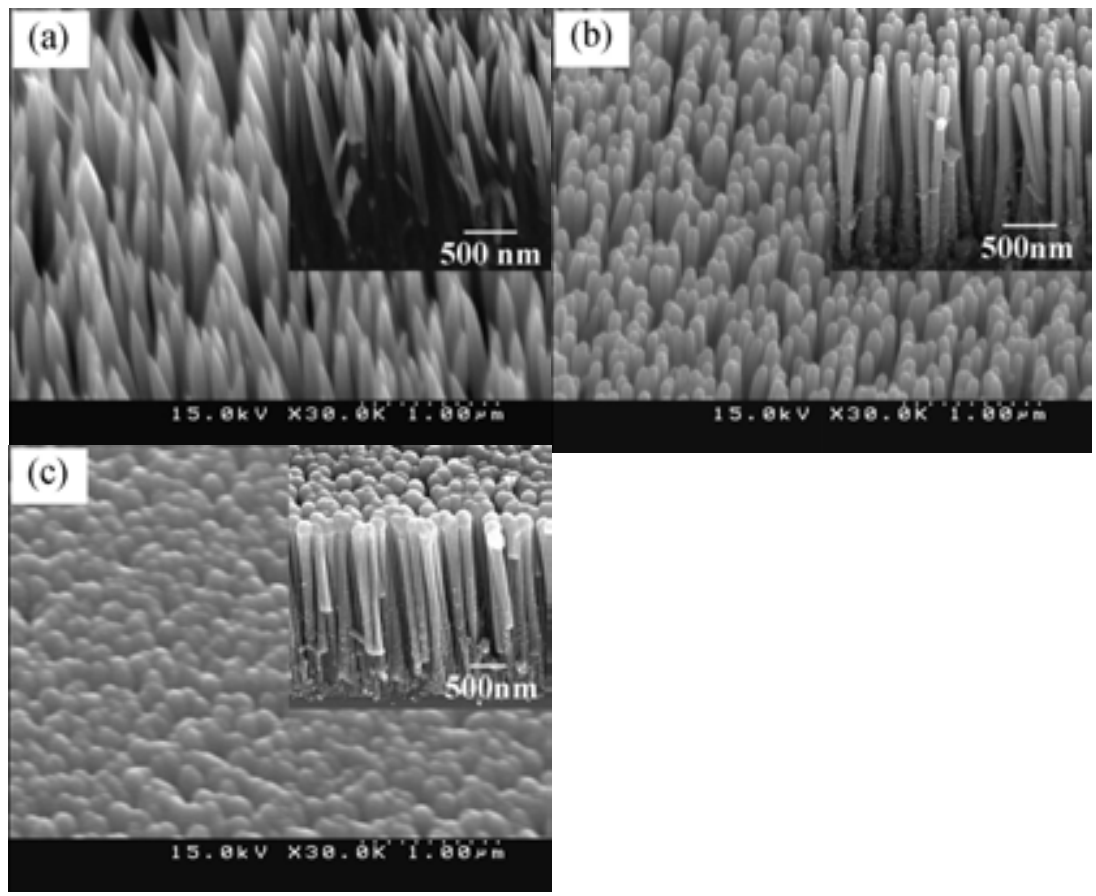

Figure 1. Surface morphologies of vertically aligned ZnO nanowires: (a) nanoneedles, (b) nanonails and (c) nanowires with rounded tip.

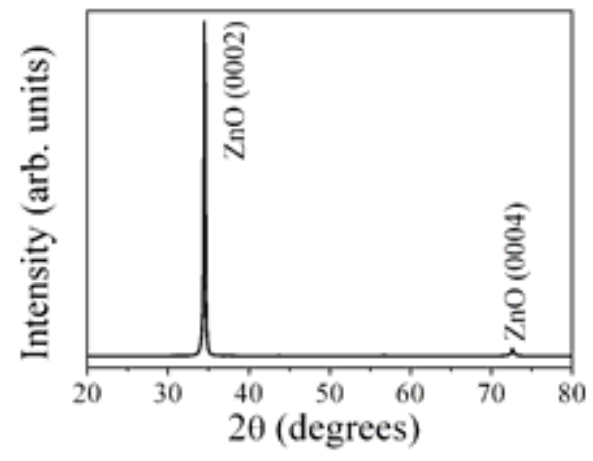

Figure 2. XRD pattern of a representative $\mathrm{ZnO}$ nanowire sample 


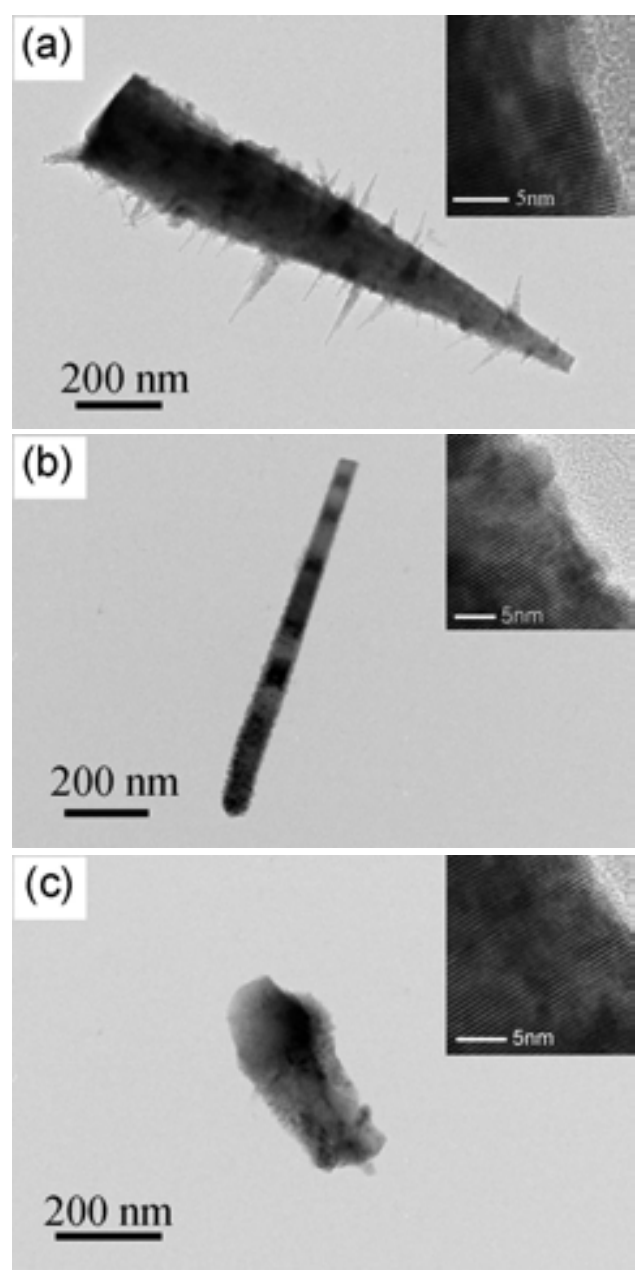

Figure 3. TEM images of ZnO NRs: (a) nanoneedles, (b) nanonails and (c) nanorods with rounded tip. Inset shows the corresponding HRTEM images

\begin{tabular}{cccccc}
\hline Sample & Substrate temp. $\left({ }^{\circ} \mathbf{C}\right)$ & $\begin{array}{l}\mathbf{O}_{2} \text { flow } \\
(\mathbf{S C C M})\end{array}$ & $\mathbf{N}_{\mathbf{2}}$ flow (SCCM) & Working Pressure (torr). & Time (min) \\
\hline (a) & 630 & 20 & 70 & 3 & 90 \\
\hline (b) & 630 & 50 & 50 & 1 & 30 \\
\hline (c) & 630 & 50 & 50 & 1.5 & 60 \\
\hline
\end{tabular}

Table 1. Growth parameter of ZnO nanorod arrays. 
To investigate the effect of lattice mismatch on the growth mechanism, $\mathrm{ZnO}$ nanowires were grown on A (1120), C (0001) and R (1012) plane sapphires using MOCVD system. Here, the working pressure of the reactor was maintained at 6 Torr and the distance between the susceptor and the nozzle was fixed at $5 \mathrm{~cm}$. Fig. 3 depicts the FESEM images of $\mathrm{ZnO}$ nanostructures on various sapphire substrates. Vertically aligned nanowires of width 100-150 nm and length 3-4 $\mu \mathrm{m}$ are grown on A- and C-plane sapphires (Fig. 4(a) and (b)), but nanopencil/tip like $\mathrm{ZnO}$ nanostructures of length 1.5-2.5 $\mu \mathrm{m}$ is observed for the R-sapphire substrate (Fig. 4(c)). The average basal diameter of the nanopencils is around 250-300 $\mathrm{nm}$ and its shape becomes narrow towards top-end. Sharp tip of the nanostructures may be due to the residual amount of precursors at the end of the growth process. For better understanding of the growth process, nanostructures were grown for a longer time (90 $\mathrm{min})$. For both A- and C-plane sapphires, the nanowires retain their vertical nature and are uniform throughout its length, which clearly indicates the growth is along c-axis (Fig. 4(d)). On the other hand, Fig. 4(e) shows a typical image of ZnO nanostructures on R-plane sapphire, in which lateral growth has been taken place indicating a deterioration of c-axis growth. This may be due to the generation of stress during the initial stage of growth and afterwards these nanostructures join together to make thicker structures. The magnified image (inset of Fig. 4(e)) revealed that the thicker nanostructures are randomly oriented and their surface is appeared to be comparatively rough. The bright-field (BF) TEM image of single $\mathrm{ZnO}$ nanowire is shown in Fig. 4 (e). This shows the diameter of the nanowires grown on A, C sapphire are around $100 \mathrm{~nm}$ and where as it is around $300 \mathrm{~nm}$ for the nanowire grown on $\mathrm{R}$ sapphire. The tip of the nanowire was gradually tapered down from several hundred of nanometers to $50 \mathrm{~nm}$. This is due to the lack of the fresh reactant supply towards the final stage of growth process. At the initial stage of the growth, the width of the nanowires were thin and the individual thin nanowires laterally merged together to give thicker one with increase in growth time. Generally, the formation mechanism of nanostructures consists of two stages: nucleation and growth. At first, nanosized crystalline nuclei are formed on the substrate and afterwards the nanowires start to grow along a specific direction. The diameter of the nanowires depends on the size and density of the initial $\mathrm{ZnO}$ nuclei. In case of C-plane sapphire, $\mathrm{ZnO}$ has a partial ionic character, which originates due to the opposite charge on the (0001) and (0001) planes being $\mathrm{Zn}$ and $\mathrm{O}$ termination, respectively [38]. Thus there is a net dipole moment when the crystal is terminated by the basal plane, which causes the surface energy to diverge. On the other hand, the (1120) plane is nonpolar as it comprises an equal number of $\mathrm{O}$ and $\mathrm{Zn}$ atoms, and hence has a lower surface energy [39-40]. Therefore the growth rates of $\mathrm{ZnO}$ nanostructures on $\mathrm{C}$ - and R-plane sapphires are different, which is confirmed from the variation in aspect ratio (length/width) of the nanostructures. Hence, it is expected that the condition of supersaturation is different for the growth of $\mathrm{ZnO}$ nanostructures on $\mathrm{C}$ and R-plane sapphires. At present, it is believed that there is a formation of an unintentional thin interfacial $\mathrm{ZnO}$ layer during the initial stage of growth on R-plane sapphire. Furthermore, the shape of the nanowires was change to nanopencil using an intentional thin $\mathrm{ZnO}$ film as an interfacial layer [41]. This indicates that the different shape, size and density of the nanowires can be attributed to the in- 
plane stress during the initial stage of growth, which comes from thermal and lattice mismatches between $\mathrm{ZnO}$ nanostructures and the substrates [42-43]. According to crystallographic point of view, Miyauchi et al. [44] reported that the probability of oxygen vacancies at the terminated surfaces of $\mathrm{ZnO}$ nanostructures grown on R-plane sapphire is higher than others. The surface morphology as well as crystallinity of $\mathrm{ZnO}$ nanostructures, grown on R-plane sapphire, is quite different from A- and C-plane sapphires. For R-plane sapphire, it is expected that simultaneous existence of $\mathrm{O}$ and $\mathrm{Zn}$ atoms in (1120) plane plays a vital role on the property of nanostructure.
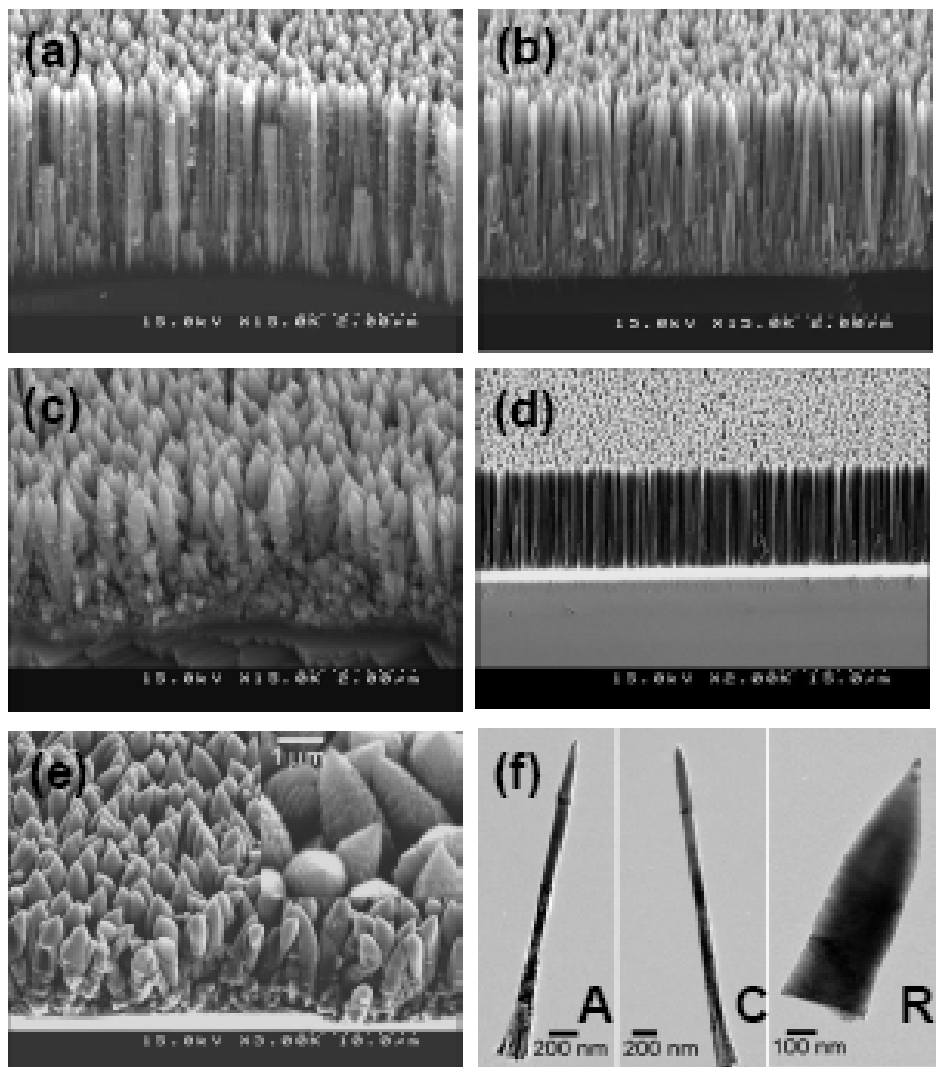

Figure 4. Micrographs of ZnO nanostructures on (a) A-, (b) C-, (c) R-plane sapphires for 15 min growth, (d) C-, (e) Rplane sapphires for 90 min growth, and (f) bright-filed TEM images [J. P. Kar et al. Applied Surface Science 256 (2010) 4995]. 


\subsection{Effect of Si Substrate}

To investigate the effect of substrate, $\mathrm{ZnO}$ nanostructures were grown on Si substrate and $\mathrm{Si}$ with $\mathrm{ZnO}$ interfacial layer. Pencil like $\mathrm{ZnO}$ nanostructures were obtained on Si substrate with $\mathrm{ZnO}$ thin film as an interfacial layer (Fig. 5). Here, the substrate temperature, working pressure and substrate to nozzle distance were kept at $600{ }^{\circ} \mathrm{C}$, 3 Torr and $3 \mathrm{~cm}$, respectively. With the decrease in substrate to nozzle distance $(\sim 1 \mathrm{~cm})$ and increase in the substrate temperature $700{ }^{\circ} \mathrm{C}$, a hallow microtube like structures were grown. FE-SEM images of the vertically aligned $\mathrm{ZnO}$ microtubes with a hollow tubular shape are shown in Fig. 6. The magnified SEM images (insets) show that all of the microtubes have a hollow cavity and a faceted hexagonal shape. The tubular wall-thickness is in the range of micrometers towards base and it reduced to nanometers range towards top-end. The average outer basal diameter of the microtubes is around $10 \mu \mathrm{m}$ and its shape becomes narrow $(5 \mu \mathrm{m})$ towards top end. The length of the $\mathrm{ZnO}$ microtubes is about $40 \mu \mathrm{m}$ and the diameter is $\sim 4 \mu \mathrm{m}$ towards the open tip whereas it becomes $1-1.5 \mu \mathrm{m}$ at the basal part. Typical crystal morphology and a representative cross-sectional view of a single $\mathrm{ZnO}$ microtube is shown in Fig. 6(c). Hollow basal part of the tubular structure (inset of Fig. 6(c)) consists of many nanospikes (width $\sim 50-120 \mathrm{~nm}$ and length $\sim 500 \mathrm{~nm}$ ). We believe that these nanospikes grow further during the course of the growth process and merge together to make solid surface, which in fact reduces the inner diameter. From XRD pattern (not shown here), a prominent $\mathrm{ZnO}$ (0002) peak is observed, which corresponds to the growth of $\mathrm{ZnO}$ microtubes along c-axis. No characteristic peaks indicating other impurities are observed in the samples. This is attributed to the hexagonal wurtzite structure of microtubes. Vertically aligned $\mathrm{ZnO}$ microtubes display inerratic or perfect hexagon-shaped cross-sectional structure and open tips. At the initial stage of growth, several vertically aligned nanowires are generated due to the high growth rate of $\mathrm{ZnO}$ crystal along [0001] direction, which is thermodynamically stable. As the growth time increases, the $\mathrm{ZnO}$ nanowires may grow laterally even though the lateral growth rate is much lower than that of the vertical direction. Moreover, a high reaction temperature may accelerate the lateral growth of the $\mathrm{ZnO}$ nanowires. Therefore, neighboring $\mathrm{ZnO}$ nanowires gradually start to coalesce together to form hexagonal circles. As the reaction time increases, the complete prismatic $\mathrm{ZnO}$ microtubes are finally formed by the coalescence of $\mathrm{ZnO}$ nanowires. Furthermore, the substrate was very close $(1 \mathrm{~cm})$ to the nozzle of the precursor, which favored the reasonable supply of precursors. It is clear that the morphology changed from the nanowires to microtubes with the in increase growth temperature and decrease in nozzle-substrate spacing. Hence, the higher growth temperature and lower nozzle-substrate spacing favor the "oriented attachment" process of the nanowires to form microtubes. At present, it is believed that substrate temperature has more influence for the quality of the microtubes. On the other hand, it has also been reported that catalysts like carbon and metastable zinc-rich oxide layer played a vital role for the generation of tubular structures $[45,46]$. 

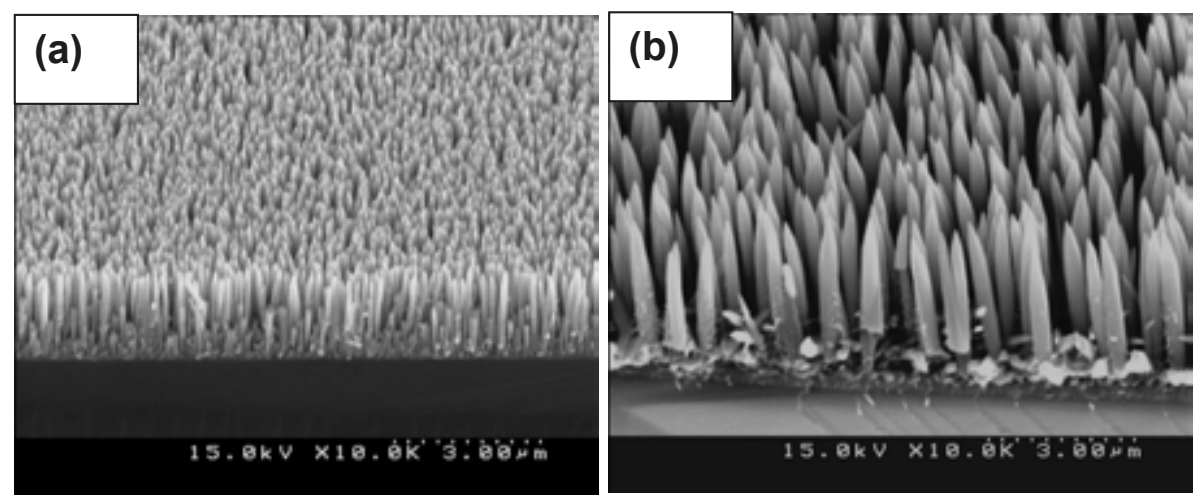

Figure 5. FE-SEM images of ZnO nanowires grown on (a) Si substrate, (b) Si substrate with ZnO interfacial layer.
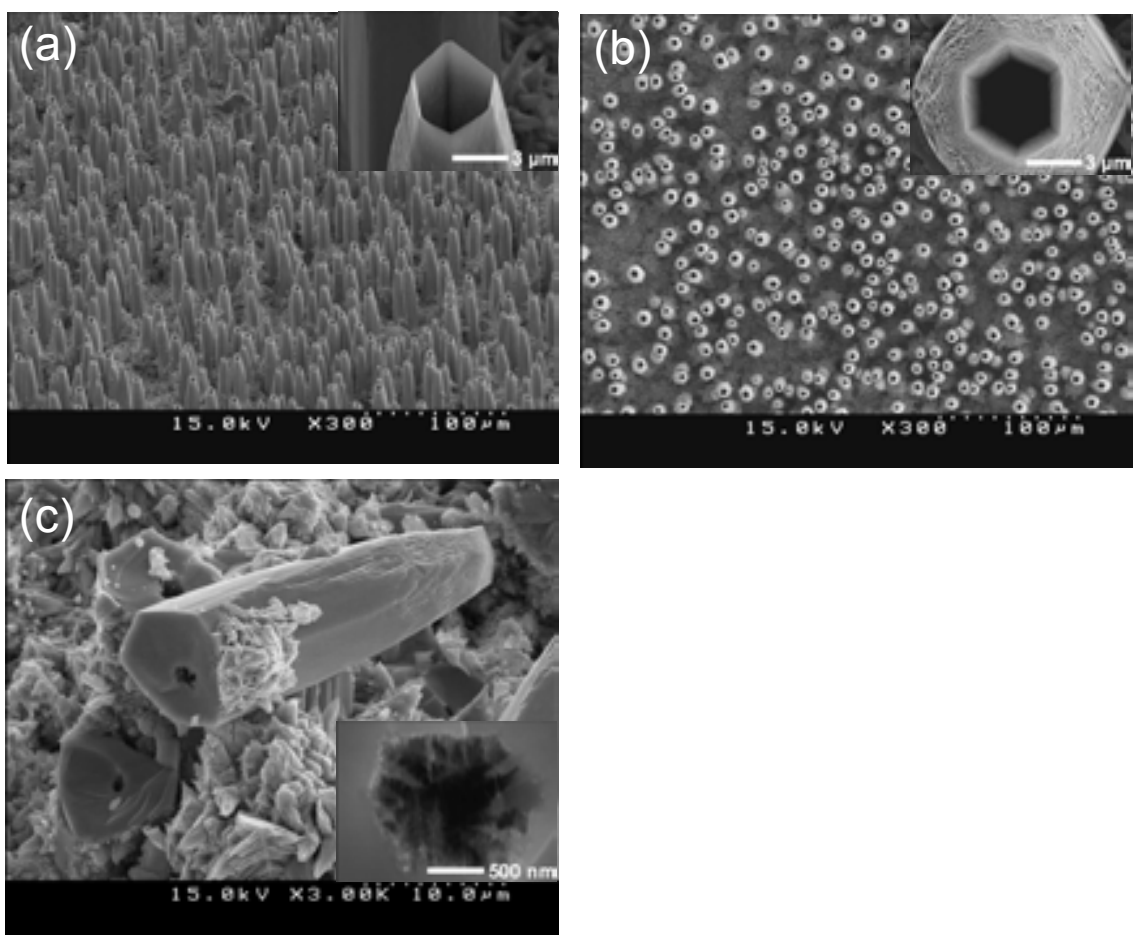

Figure 6. FE-SEM (a) $45^{\circ}$ tilted, (b) top, and (c) cross-sectional images of ZnO microtubes grown on Si substrates [J. P. Kar et al. Materials Letters 63 (2009) 2327]. 
To investigate the effect of the shape of Si substrate, $\mathrm{ZnO}$ nanostructures were grown by MOCVD technique on Si microchanel. Before $\mathrm{ZnO}$ growth, Si substrates were patterned by wet chemical etching process to form microcavity. At first, 200-nm-thick silicon nitride masking layer was grown by low-pressure chemical vapor deposition (LPCVD) at $800{ }^{\circ} \mathrm{C}$ in the mixture of di-chloro silane $\left(\mathrm{SiH}_{2} \mathrm{Cl}_{2}\right)$ and ammonia $\left(\mathrm{NH}_{3}\right)$ with 240 mTorr pressure.Thereafter, standard photolithography and etching techniques were adopted in order to fabricate micropatterns. Reactive ion etcher (RIE) was used to remove silicon nitride layer from the micro- patterned windows and the etch rate was $350 \mathrm{~nm} / \mathrm{min}$. Afterwards, the samples were immersed in $\mathrm{KOH}$ solution for 10 hour to fabricate the truncated pyramid-shaped etch patterns. Before the growth of $\mathrm{ZnO}$ nanowires, no passivation layer was intentionally used. The substrate temperature was kept at $700{ }^{\circ} \mathrm{C}$ during the nanowire growth. The working pressure of the reactor was maintained at 3 Torr and the distance between the susceptor and the nozzle was fixed at $1.5 \mathrm{~cm}$. Fig. 7 and Fig. 8 show the crosssectional image (FESEM) of the different microchannel in silicon substrate, which contains $\mathrm{ZnO}$ nanowires. Three parts (top surface [Fig. 7(a), (b) and Fig. 8(a) and (b)], side wall [Fig. 7(c) and Fig. 8(c)] and bottom surface [Fig. 7(d), (e) and Fig. 8(d) and (e)]) have $\mathrm{ZnO}$ nanowires with different dimensions and surface morphologies. On the top of silicon substrate the nanowires were almost uniform along the length $(2 \mathrm{~mm})$ and width of 200-250 nm. The aspect ratio, packing fraction and the number density of nanowires on top surface are around 10, 0.8 and $10^{7}$ per $\mathrm{mm}^{2}$, respectively. The packing fractions of nanowires were determined by measuring the area occupied by the nanowires on a top view micrograph. Interestingly, vertical nanopencil /tip like $\mathrm{ZnO}$ nanostructure was observed towards the bottom of the trench. The $\mathrm{ZnO}$ nanowires have preferentially grown along a particular direction, which his normal to the substrate surface. It is expected that at the bottom surface the nanowires would have a high perpendicular anisotropy because of the high aspect ratio and the low packing fraction. Figs. 7(e) show the microstructures of $\mathrm{ZnO}$ nanowires at the bottom corner of the microchannel. Furthermore, randomly distributed few nanoflowers are also observed at side wall sand bottom of the microchannel. It may be generated from the defects of Si surface, arrived during etching of silicon. The flower has several wings, which have grown from the common origin. These happen due to the different directional growth of $\mathrm{ZnO}$ nanowires at the defect points. The possible growth mechanisms of $\mathrm{ZnO}$ nanowires are stated below. At the top surface, high-density island were nucleated on the substrate and grew preferentially along the c-axis orientation. The continuous growth led to the coalescence of these islands and as a result wider nanowires, with a low density and a reduced aspect ratio, were grown. But at the inclined surface, the growth direction (c-axis) and the direction of incoming precursor molecules are not same.

So, the width of nanowires is less than the top surface. Now at the bottom surface of the microchannel, number of incoming fresh precursor molecule is less than the top surface due to stagnant of gaseous species at the cavity. It is assumed that the sharpness of the tips originates due to the lack of fresh reactant supply to the bottom of cavity during the growth of nanostructures. Thus, the probability of coalescence of grain is less, which leads to the formation of thin nanowires compared to the top surface. 

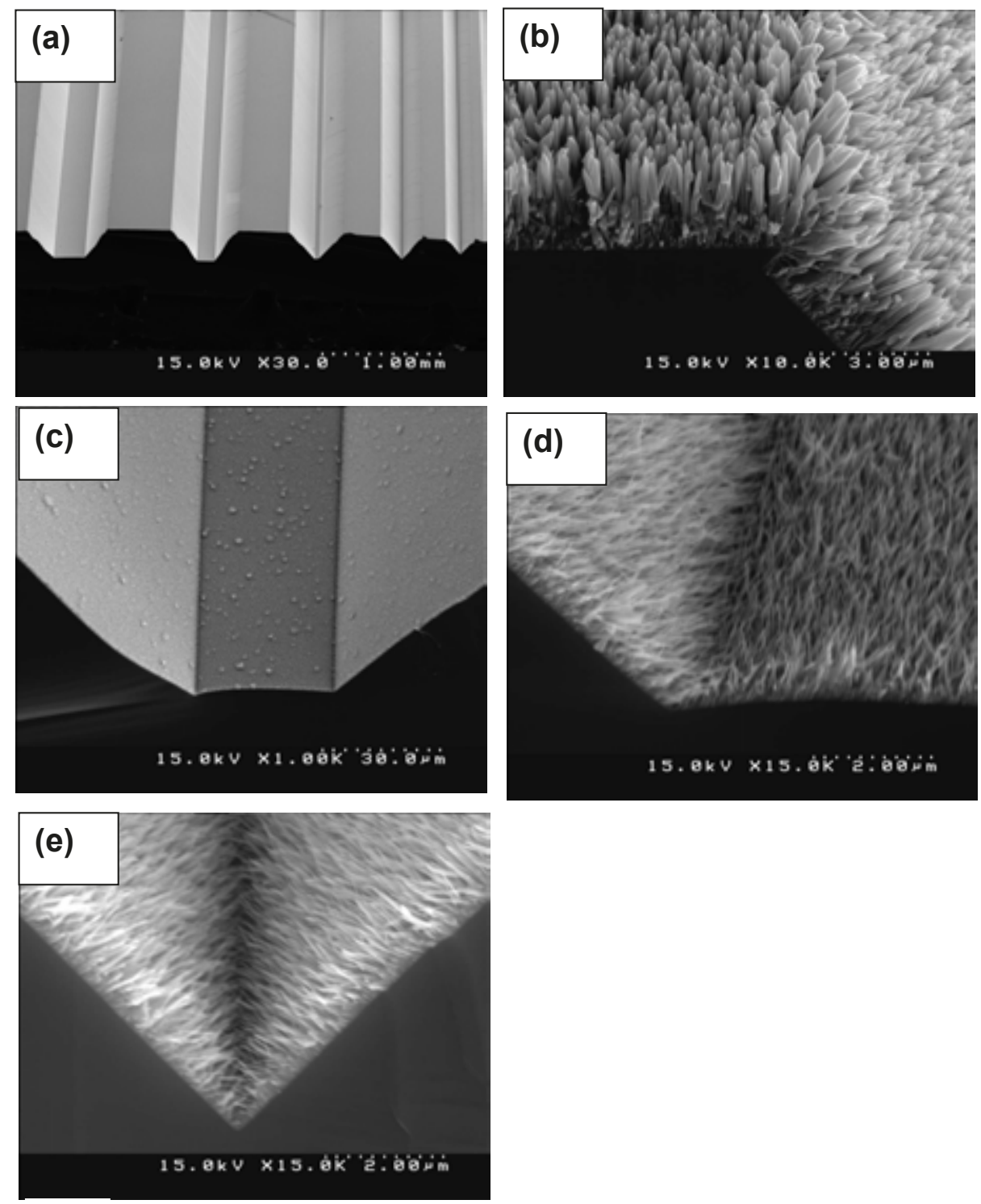

Figure 7. FESEM images of ZnO nanowires on (a) microchannel, (b) Top surface, (c-e) magnified image of bottom surface. 

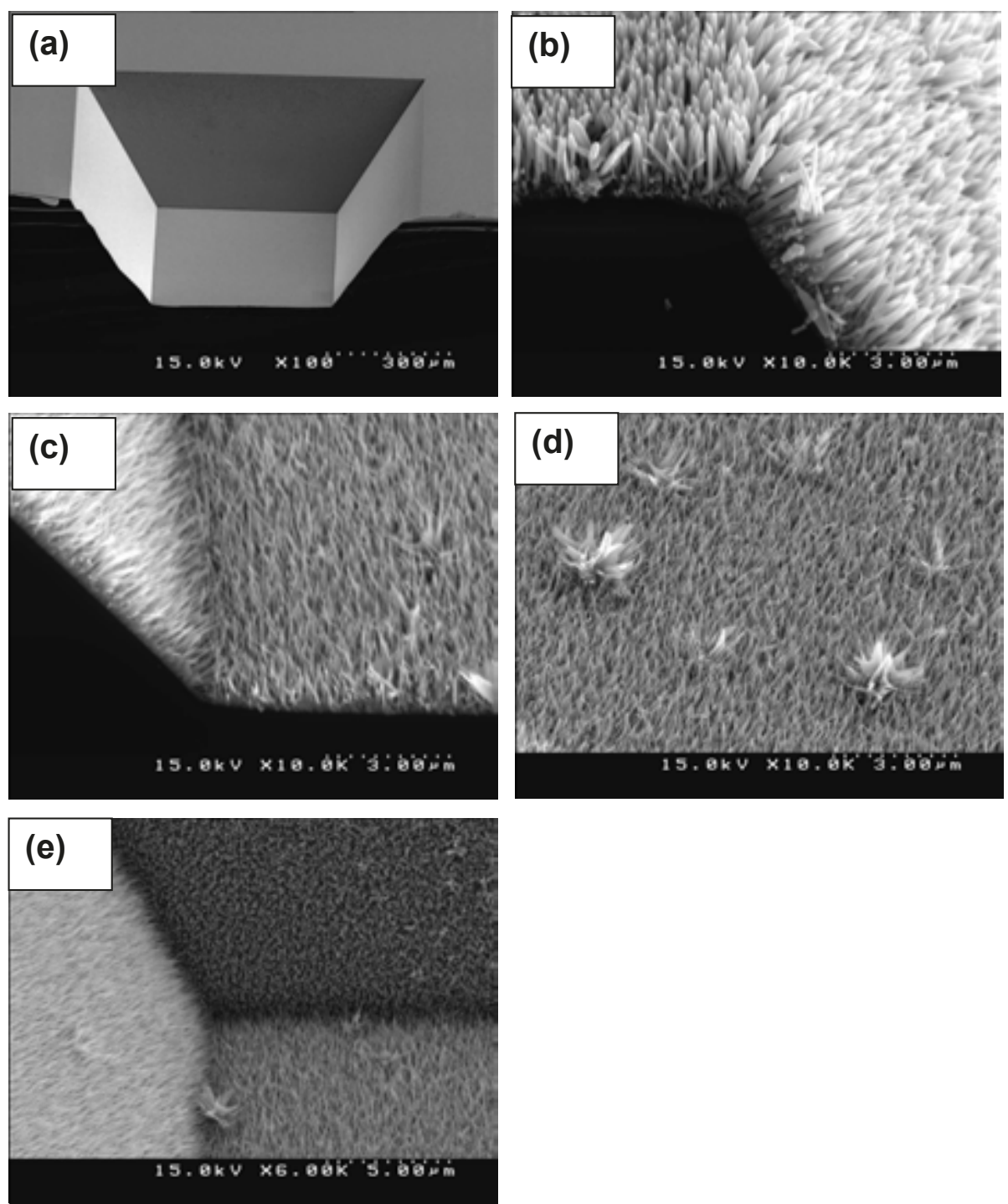

Figure 8. FESEM images of ZnO nanowires on (a) microchannel, (b) Top surface, (c) bottom surface, (d) magnified image of bottom surface, (e) bottom corner.

\subsection{Effect of interfacial layer}

To investigate the effect of interfacial layer on $\mathrm{ZnO}$ growth by MOCVD technique, Ga doped $\mathrm{ZnO}$ coated glass and $\mathrm{ZnO}$ microcators were used as substrates. Ga-doped $\mathrm{ZnO}$ films were deposited by DC sputtering of GZO target on corning glass (1737) substrates. The sub- 
strate temperature, working pressure and sputtering power were fixed around $280{ }^{\circ} \mathrm{C}, 3 \times 10^{-3}$ torr and $1.2 \mathrm{~kW}$, respectively. The substrate temperature and working pressure were fixed at $600{ }^{\circ} \mathrm{C}$, 3 Torr and $1.5 \mathrm{~cm}$, respectively. The evolution of columnar NWs were possible when the flow rate of $\mathrm{O}_{2}(20 \mathrm{sccm})$ was lower than the carrier Ar gas $(50 \mathrm{sccm})$.

Vertical aligned long $\mathrm{ZnO}$ nanowires (width 400-500 nm) were then grown on GZO coated glass substrates and the FESEM image is shown in Fig. 9(b). In order to compare, ZnO nanowires were also grown on c-sapphire substrates keeping all of the parameters same (Fig. 9(a)). This observation depicts the lengths $(\sim 25 \mu \mathrm{m})$ of the nanowires are similar for both the substrates. These kind of long nanowires are suitable for the single nanowire based devices, where the larger length is feasible for various electrical contacts. XRD patterns (not shown here) of $\mathrm{ZnO}$ nanowires, grown on $\mathrm{GZO} /$ glass substrate shows the presence of (0002) and (0004) peaks. The absence of additional peaks in the XRD pattern excludes the possibility of any extra phases. The nanowires exhibited a prominent (0002) peak with full width at half maximum (FWHM) values around 0.06 , indicating high crystallinity of the $\mathrm{ZnO}$ nanowires. These data reveal that the $\mathrm{ZnO}$ nanowires are all c-axis oriented with wurtzite structure. The bright-field (BF) TEM image of single $\mathrm{ZnO}$ nanowire is shown in Fig. 10 (a). This shows the diameter of the nanowires is $460 \mathrm{~nm}$ without any side branches. The tip of the nanowire was gradually tapered down from several hundred of nanometers to $50 \mathrm{~nm}$. This is due to the lack of the fresh reactant supply towards the final stage of growth process. The dopant redistribution at high process temperature is a usual phenomenon during the fabrication of semiconductor devices. Herein, the nanowires were grown at $600{ }^{\circ} \mathrm{C}$ and hence there may be a chance of dopant diffusion at the interfacial layer. In this regard, cross-sectional TEM as well as EDX spectroscopic measurements of the $\mathrm{ZnO}$ nanowires on GZO film are carried out. Figure 10 (a) shows the enlarged image of the interface between the $\mathrm{ZnO}$ nanowires GZO films. This enlarged image indicates that the sputtered film has columnar grains and the nanowires are grown along the columnar grains of the GZO film.

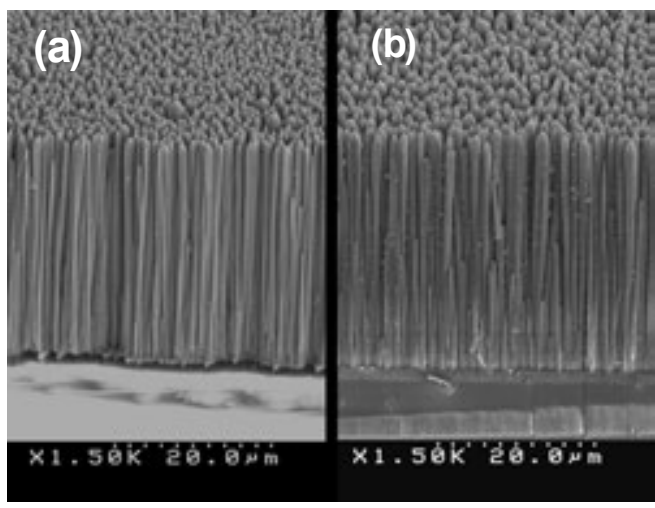

Figure 9. FESEM of ZnO NWs on (a) c-plane sapphire. (b) GZO/glass substrates 

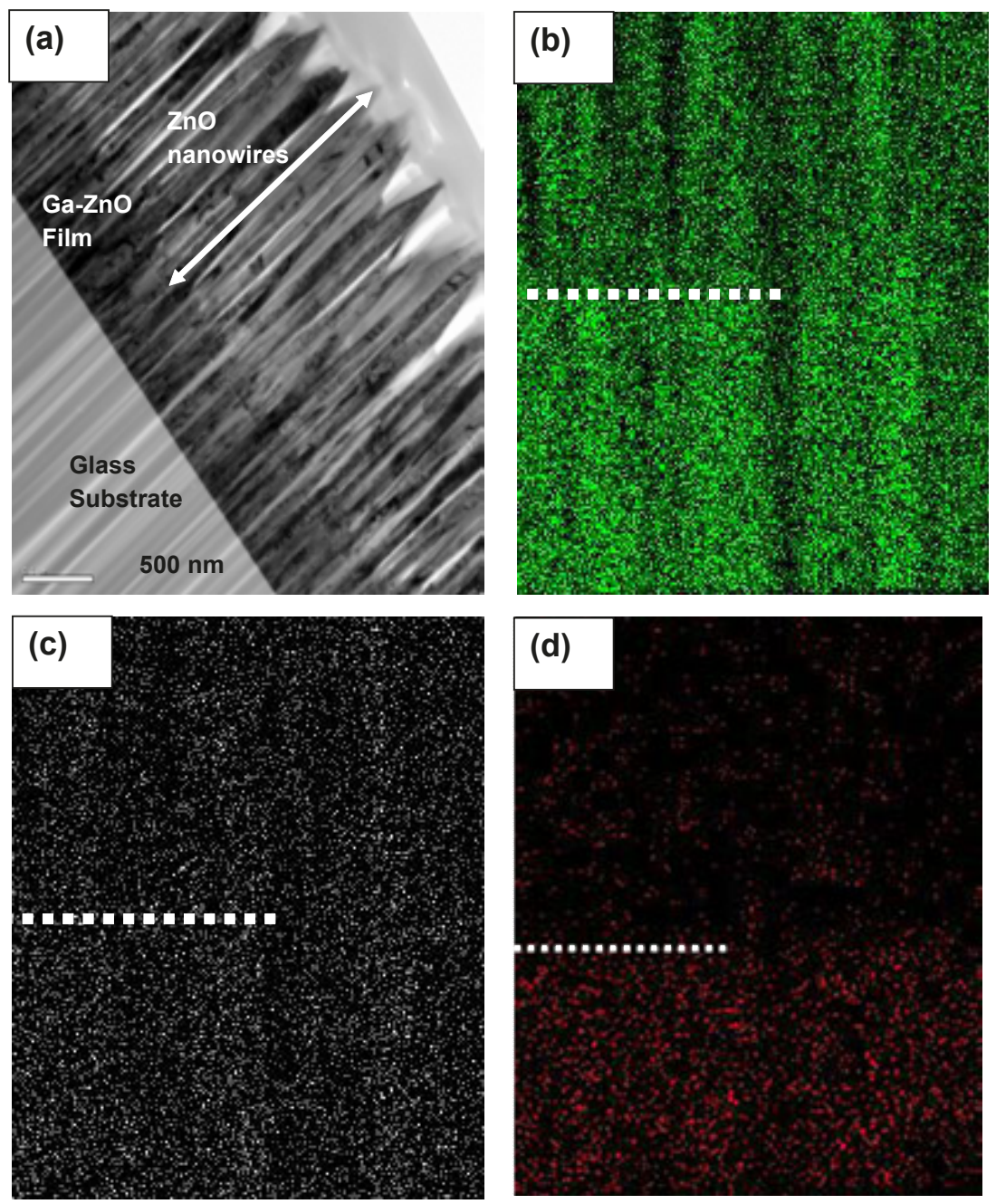

Figure 10. a) Cross-sectional TEM image across the interface of ZnO NWs and GZO film, and EDX spectroscopic mapping images of (a) Zn, (b) O and (c) Ga across the interface.

The mapping images Fig. 10(b-c), of $\mathrm{Zn}$ and $\mathrm{O}$ atoms were distributed uniformly at the interface, whereas nonuniform distributions of the Ga atoms are clearly observed (Fig.10 (d)). The Ga atoms are appeared not only in the underlying film, but also seen towards the bottom of the nanowires. The Ga concentration in the nanowires decreases with increase in distance from the interface. This observation indicates that the growth temperature provided sufficient thermal energy to Ga atoms in the interfacial film to be diffused into the lower part of nanowires. The concentration of Ga is estimated as 2.95 at. $\%$ and 0.32 at. $\%$ for GZO 
film and the bottom of the $\mathrm{ZnO}$ nanowires, respectively. The lightly incorporation of Ga towards the bottom of the nanowires, during the initial stage of growth, does not alter the columnar growth due to the similar atomic radius of $\mathrm{Ga}$ and $\mathrm{Zn}$ as well as the comparable covalent bond lengths of Ga-O and $\mathrm{Zn}-\mathrm{O}$. Yan et al. observed the evolution of nanostructures with $\mathrm{Ga}$ doping in contrast to undoped $\mathrm{ZnO}$, where Stranski-Krastanov (SK) model was used to explain the phenomena [47]. We have already observed that undoped ZnO film on Si substrate favours the formation of low density, thick nano-pencil structure by MOCVD technique. Thus Ga has an ability to scale down the grain size of the GZO film, which stimulates the formation of large number of homogeneous nucleation sites at the initial stage of nanowires growth. The c-axis orientation in $\mathrm{ZnO}$ nanowires can be explained by the "survival of the fastest", where the nuclei were formed with various orientations at the initial stage of the deposition and each nucleus competed to grow but only the nuclei in c-axis orientations could survive due to the fastest growth rates. Once the nanowires have grown, the high-speed laminar gas flow under our specific growth parameters induces turbulent flow between the nanostructures, which results in adsorption of fresh reactant gases only on nanowires tips. Hence the growth of very long nanowires is achieved. It has been reported that beyond a certain doping level $\mathrm{ZnO}$ nanowires retains its vertical shape and above this the tip of the nanostructures bends due to the generation of stacking faults and the surface becomes very rough. In the present case, such kind of abnormality has not been seen because of the mild doping of $\mathrm{Ga}$ at the bottom of the nanowires during the initial growth.

To study the effect of interfacial layer as well as the shape of the substrate, $\mathrm{ZnO}$ micro-craters were fabricated. To do so, AZO thin films were deposited on Corning glass (1737) by pulsed DC sputtering of an AZO ceramic target ( $\mathrm{Al} \sim 2 \mathrm{wt} . \%)$ with $\operatorname{Ar}(5 \mathrm{~N})$ as the ambient gas. The sputtering chamber was evacuated to a base pressure of about $5 \times 10^{-5}$ Torr. Prior to the sputtering, the target was pre-sputtered to remove any residual contamination from the target surface. The deposition parameters, pulse frequency $(2 \mathrm{kHz})$, DC power $(2 \mathrm{KW})$, substrate temperature $\left(200{ }^{\circ} \mathrm{C}\right)$, working pressure $(3 \mathrm{mTorr})$ and deposition time $(10 \mathrm{~min})$, were optimized and kept constant for all the films. Then the AZO films were anisotropically etched for $90 \mathrm{~s}$ by dilute hydrochloric acid $\left(0.5 \% \mathrm{HCl}\right.$ in $\left.\mathrm{H}_{2} \mathrm{O}\right)$ in order to create textured surfaces. Fig. $11(\mathrm{a}-\mathrm{b})$ shows the $45^{\circ}$ tilted FESEM images of the as-deposited film and the AZO films etched for $90 \mathrm{~s}$. Before etching, the film is very smooth (Fig. 11(a)). After etching, hexagonal, conical craters with diameters of $0.5-2.5 \mathrm{~lm}$ are created on the film surface (Fig. 11(b)). The formation of craters mainly occurs at positions of O-terminated $\mathrm{ZnO}$ columns, as it is highly sensitive to acid attack. The exposed facets of the etched surfaces may mainly be $\left(\begin{array}{lll}1 & 0 & 1\end{array}\right)$ plane [48]. Thereafter, the etched AZO films were put into a metal-organic chemical vapor deposition (MOCVD) chamber for growth of $\mathrm{ZnO}$ nanowires. During the growth period, the substrate temperature, working pressure and the nozzle-substrate spacing were fixed at $550{ }^{\circ} \mathrm{C}, 3$ Torr and $1 \mathrm{~cm}$, respectively. The deposition time was $5 \mathrm{~min}$. $\mathrm{ZnO}$ nanowires onto its surface to fabricate the hierarchical structure. Fig. 11(c) shows the SEM images of the hierarchical structure of the $\mathrm{ZnO}$ nanowires. All surfaces of the craters are covered by nanowires with cone-shaped tips, $50-100 \mathrm{~nm}$ in diameter and $150-300 \mathrm{~nm}$ in length. The structure as shown is very similar to that of lotus leaf. The lotus leaf surface is a good example of how, the combination of a micro-/nano-hierarchical structure and low surface energy wax layer, exhibits high water con- 

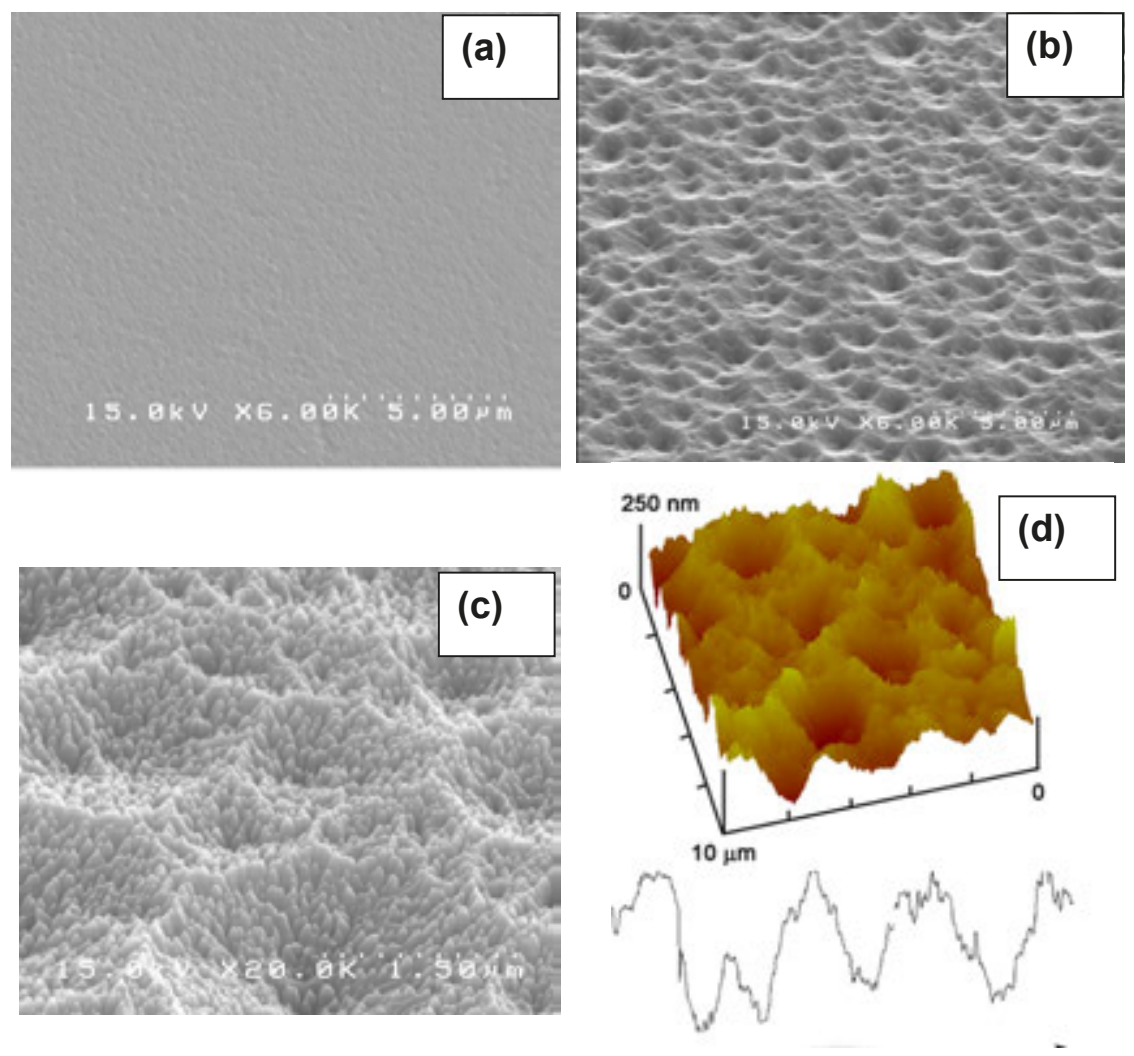

Figure 11. FESEM images ( $45^{\circ}$ view) of the AZO films: (a) as-deposited, (b) etched for 90 seconds (c) nanowires on etched sample. (d) AFM image with cross section profile (J. Xiong et al J. Colloid and Interface Science 350 (2010) 344.).

tact angle (CA) and low CA hysteresis. A superhydrophobic and self-cleaning surface mimics the surface of a lotus leaf. The combination of both self-cleaning and antireflective properties is very desirable for applications in outdoor photovoltaic and display devices, self-cleaning windows and car windshields. A two step method, using a combination of top down and bottom-up approaches, for fabrication of $\mathrm{ZnO}$ based hierarchical structures with nanowires on microcraters helps to mimic the lotus leaf structure. Fig. 11(d) shows the AFM image with the corresponding typical cross sectional profile of the etched films after the growth of nanowires. The microcraters can be easily recognized by the hexagonal holes on the surface. Due to the co-existence of micro- and nano-scale features and the non-vertical geometric alignment, the structure can induce multiple scattering of the incident light. The co-existence of microand nano-structures can interact with light of different wavelengths, causing reflective suppression over a wide wavelength range [49]. Another advantage of the hierarchical structure over flat surface film having an antireflection property that is less angle dependent. This indicates a potential application in developing Si thin film solar cell, where it can act as a front 
electrode (replacing ITO, etched AZO or textured FTO film) to achieve improved light trapping. Furthermore, it can also be used in the dye-sensitized solar cell as an electrode for the collection of electrons. The hierarchical structure can trap light, improve dye absorption and introduce a nano-scale pathway for carrier transportation, similar with the flower-like $\mathrm{ZnO}$ structure. Similarly, this structure can also be used for enhancing the extraction efficiency of LED, which is usually realized by using photonic crystals, conductive omnidirectional reflectors, or surface roughening of transparent oxide electrodes (normally ITO).

\section{Conclusion}

Among the different synthesis process, MOCVD is superior to reproduce large area; catalytic free, long, vertically aligned $\mathrm{ZnO}$ NWs with excellent morphological, structural and optical properties. As the growth of $\mathrm{ZnO}$ nanowires by MOCVD is a bottom-up technique, the nature of substrate, shape of the substrate and interfacial layer on the substrate have vital role for the determination of nanowire dimension and alignment. According to lattice misfit, the most suitable substrate for $\mathrm{ZnO}$ growth is $\mathrm{ScAlMgO}_{4}$, which is expensive and technologically inconvenient. Generally, the formation mechanism of nanostructures consists of two stages: nucleation and growth. At first, nanosized crystalline nuclei are formed on the substrate and afterwards the nanowires start to grow along a specific direction. The c-axis orientation in $\mathrm{ZnO}$ nanowires can be explained by the "survival of the fastest", where depending on the nature of the substrate, the nuclei were formed with various orientations at the initial stage of the deposition and each nucleus competed to grow but only the nuclei in c-axis orientations could survive due to the fastest growth rates. Once the nanowires have grown, the high-speed laminar gas flow under our specific growth parameters induces turbulent flow between the nanostructures, which results in adsorption of fresh reactant gases only on nanowires tips. Hence the growth of very long nanowires is achieved. At the initial stage of the growth, the width of the nanowires were thin and the individual thin nanowires laterally merged together to give thicker one with increase in growth time. Thus, the diameter of the nanowires depends on the size and density of the initial $\mathrm{ZnO}$ nuclei. Also the supply of the fresh reactant during the growth process takes a vital role on the nanostructure. Among different substrates, nanowires on C-plane sapphire show the excellent optical and structural properties. On the other hand, by changing the deposition condition of MOCVD and type of substrate one can synthesize $\mathrm{ZnO}$ nanowire of desired structure for different application.

\section{Acknowledgements}

This research was supported by WCU (World Class University) program through the National Research Foundation of Korea funded by the Ministry of Education, Science and Technology (R32-20031). 


\section{Author details}

Sachindra Nath Das ${ }^{1,3}$, Jyoti Prakash Kar ${ }^{2,3}$, Junjie Xiong ${ }^{3}$ and Jae-Min Myoung ${ }^{3}$

1 Department of Physics, Burdwan Raj College, Burdwan, West Bengal, India

2 Department of Physics, National Institute of Technology, Rourkela, Odisha, India

3 Department of Material Science and Engineering, Yonsei University, Seoul, South Korea

\section{References}

[1] Pan Z.W., Dai Z.R., Wang Z.L. Science 2001; 291: 1947.

[2] Lee C.J., Lee T.J., Lyu S.C., Zhang Y., Ruh H., Lee H.J. Appl. Phys. Lett 2002; 81: 3648.

[3] Zhao Q., Zhang H.Z., Zhu Y.W., Feng S.Q., Sun X.C., Xu J., Yu D.P. Appl. Phys. Lett. 2005; 86: 203115.

[4] Xu C.X., Sun X.W. Appl. Phys. Lett. 2003; 83: 3806.

[5] Gao P.X., Wang Z.L. Small 2005; 1: 945.

[6] Yan H., He R., Johnson J., Law M., Saykally R.J., Yang P. J. Am. Chem. Soc. 2003; 125: 4728.

[7] Xu J., Pan Q., Shun Y., Tian Z., Sens. Actuators B 2000; 66: 277.

[8] Cheng X.L., Zhao H., Huo L.H., Gao S., Zhao J.G., Sens. Actuators B 2004;102: 248.

[9] Xiangfeng C., Dongli J., Djurišic A.B., Leung Y.H., Chem. Phys. Lett. 2005; 401: 426.

[10] Wan Q., Li Q.H., Chen Y.J., Wang T.H., He X.L., Li J.P., Lin C.L., Appl. Phys. Lett. 2004; 84: 3654.

[11] Liao L., Zhang W.F., Lu H.B., Li J.C., Wang D.F., Liu C., Fu D.J., Nanotechnology, 2007;18: 225.

[12] Huang Y., Yu K., Zhu Z., Curr. Appl. Phys. 2007; 7: 702.

[13] Park S., Kim S., Han S., Nanotechnology 2007; 18: 055608.

[14] Wu L., Wu Y., Pan X., Kong F., Optical Mater. 2006; 28: 418.

[15] Mahalingam T., Lee K.M., Park K.H., Lee S., Ahn Y., Park J.Y., Koh K.H., Nanotechnology 2007; 18: 035606.

[16] Kang H.S., Kang J.S., Kim J.W., Lee S.Y., Appl. Phys. Lett. 2004; 95: 1246.

[17] Leung Y.H., Djurišić A.B., Gao J., Xie M.H., Wei Z.F., Xu S.J., Chan W.K., Chem. Phys. Lett. 2004; 394: 452. 
[18] Li Y., Liu F., Sun J., Chem. Commun. 2009; 2730.

[19] Cao M., Song X., Zhai J., Wang J., Wang Y., J. Phys. Chem. B 2006; 110: 13072.

[20] Zhang X., Fujishima A., Jin M., Emeline A.V., Murakami T., J. Phys. Chem. B 2006; 110: 25142.

[21] Lee, W., Jeong, M. C., and Myoung, J. M. Acta Mater. 2004; 52: 3949.

[22] Li, S. Y., Lin, P., Lee, C. Y., and Tseng, T. Y. J. Appl. Phys. 2004; 95(7): 3711.

[23] Sun, Y., Fuge, G. M., and Ashfold, M. N. R. Chem. Phys. Lett. 2004; 396: 21.

[24] Wang, M., Ye, C. H., Zhang, Y., Wang, H. X., Zeng, X. Y., and Zhang, L. D. J. Mater. Sci. Mater. Electron. 2008; 19(3): 211.

[25] Vayssieres, L. Adv. Mater. 2003;15(5): 464.

[26] Gao, X. D., Li, X. M., Zhang, S., Yu, W. D., and Qiu, J. J., J. Mater. Res. 2007; 22(7): 1815.

[27] Hsu H.C., Cheng C.S., Chang C.C., Yang S., Chang C.S., Hsieh W.F., Nanotechnology 2005;16: 297.

[28] Ievtushenko A., Karpyna V., Lashkarev G., Lazorenko V., Baturin V., Karpenko A., Lunika M., Danko A., Acta Phys. Pol. A 2008;114: 1131.

[29] Emanetoglu N.W., Patounakis G., Liang S., Gorla C.R., Wittstruck R., Lu Y., IEEE

[30] Trans. Ultrason. Ferroelectr. Freq. Control 2001; 48 (5):1389.

[31] Wang W.S., Wu T.T., Chou T.H., Chen Y.Y., Nanotechnology 2009; 20: 135503.

[32] Sadek A.Z., Wlodarski W., Li Y.X., Yu W., Li X., Yu X., Kalantar-zadeh K., Thin Solid Films 2007; 515: 8705.

[33] Ievtushenko A., Karpyna V., Lashkarev G., Lazorenko V., Baturin V., Karpenko A., Lunika M., Danko A., Acta Phys. Pol. A 2008; 114: 1131.

[34] Liou S.C., Hsiao C.S., Chen S.Y., J. Cryst. Growth 2005; 274:438.

[35] Kim Y.S., Ha S.C., Kim K., Yang H., Choi S.Y., Kim Y.T., Park J.T., Lee C.H., Choi J., Paek J., Lee K., Appl.Phys.Lett. 2005; 86: 213105.

[36] Rasmussen A., Gaitan M., Locascio L.E., Zaghloul M.E., J.Microelectromech. Syst. 2001; 10: 286.

[37] Sanchez J.B., Berger F., Fromm M., Nadal M.H., SensorsActuatorsB 2006; 119: 227.

[38] Fang F., Zhao D.X., Zhang J.Y., Shen D.Z., Lu M., Fan X.W., Li B.H., Wang X.H., Nanotechnology 2007; 18: 235604.

[39] Miyauchi M., Shimai A., Tsuru Y., J. Phys. Chem. B 2005; 109: 13307.

[40] Zhang Y., Du G., Zhu H., Hou C., Huang K., Yang S., Opt. Mater. 2004; 27: 399.

[41] Miyauchi M., Shimai A., Tsuru Y., J. Phys. Chem. B 2005; 109: 13307. 
[42] Kar J.P., Lee S.W, Lee W., Myoung J.M., Appl. Surf. Sci. 2008; 254: 6677.

[43] Kar J. P., Das S. N., Choi J. H., Lee T. I., Myoung J. M., Appl. Surf. Sci. 2010; 256: 4995.

[44] Zhu Q.S., Wang Z.G., Appl. Phys. Lett. 2005; 87: 231903

[45] Miyauchi M., Shimai A., Tsuru Y., J. Phys. Chem. B 2005; 109: 13307.

[46] Sun T, Qiu J. Mater Lett 2008; 62:1528.

[47] Xu W.Z, Ye Z.Z, Ma D.W, Lu H.M, Zhu L.P, Zhao B.H, Appl Phys Lett 2005;87:093110.

[48] Li M.K, Wang D.Z, Shi F., Ding S., Hong J. Chin Phys Lett 2007;24:236-9.

[49] Ellmer K., Klein A., Rech B., Transparent Conductive Zinc Oxide, Springer, 2007.

[50] Zhang L., Zhang X., Dai Z., Wu J., Zhao N., Xu J., J. Colloid Interface Sci. 2010;345: 116. 